\title{
Annely ROTHKEGEL ${ }^{1}$
}

\section{RISIKO UND RESILIENZ IM RAHMEN DER NACHHALTIGKEITS-KOMMUNIKATION: SEMANTISCHE MODELLE IM UMGANG MIT KRISEN}

Der vorliegende Beitrag versucht, anhand ausgewählter theoretischer Modelle zu den Begriffen Risiko und Resilienz einige grundlegende Linien durch das semantische Terrain von Nachhaltigkeitsdiskursen zu ziehen. Den Ausgangspunkt bilden thematische Analysen von authentischen Texten (print und online) im Hinblick auf Gebrauchsweisen und Bedeutungen der Begriffe von Risiko und Resilienz, die einerseits die Gefahrenaspekte, andererseits mögliche Bewältigungsstrategien in Krisen kennzeichnen. Die NachhaltigkeitsKommunikation ist geprägt durch eine Verschränkung von Themen aus unterschiedlichen Disziplinen und den Mix von Fach- und Alltagssprache. Experten und Nicht-Experten sind beteiligt an der Lösung von Sachproblemen (z.B. Finanzkrise, Energiewende, Erhalt der Biodiversität, Klimawandel), die sich mit Kommunikationsproblemen überlagern. Letzteres bedeutet eine Herausforderung für die linguistische Diskursanalyse und stellt ein Desiderat für die theoretische und empirische Forschung dar.

Anhand ausgewählter Risiko- bzw. Resilienzmodelle wird exemplarisch gezeigt, wie die Identifikation von semantischen Modellen im Sinne unterschiedlicher Verstehens- bzw. Interpretationsmuster mögliche Verständigungsprobleme aufzudecken hilft. Insofern als ein zentraler Teil der Nachhaltigkeits-Kommunikation als Krisen-Prävention verstanden wird, wurden die Fachbegriffe Risiko und Resilienz ausgewählt, die in der Alltagskommunikation wohl nicht unbekannt, aber doch relativ unüblich sind.

Die Modelle der Risikokommunikation sind im Wesentlichen an einzelne Disziplinen gebunden, (z.B. Risiko in der Perspektive von Gefahr/Gefährdung, Schaden/Verlust, Sicherheit, Wagnis/Gewinn). Dies gilt nur teilweise für den Begriff der Resilienz (z.B. Widerstandsfähigkeit, Regenerationsfähigkeit, psychische Stabilität, Robustheit (technische Systeme)). Hier haben sich allgemeine Zuordnungen herauskristallisiert, die einem übergeordneten Modell von Gesundheit (im weitesten Sinne) entsprechen. Während die Risikomodelle vorrangig den gesamtgesellschaftlichen Rahmen der Nachhaltigkeitskommunikation abdecken, wird mit den Resilienzmodellen dem Individuum Rechnung getragen.

Schlüsselbegriffe: Risiko, Resilienz, semantische Analyse, öffentliche Kommunikation, nachhaltige Entwicklung, Krisenmanagement

\section{FRAGESTELLUNG}

Die Verbindung von Sachbezug und Sprachgebrauch prägt die linguistische Richtung der Fachkommunikation (Schubert 2007, Rothkegel 2010a). In unserem Ansatz gehen wir

\footnotetext{
${ }^{1}$ Prof. Dr. Annely Rothkegel, Gastprofessur am Institut für Übersetzungswissenschaft und Fachkommunikation Stiftung Universität Hildesheim (Bühler-Campus), Postadresse: Marienburger Platz 22, D 31141 Hildesheim, tel. 0049 (0)681 32189, e-mail: annely.rothkegel@uni-hildesheim.de
} 
davon aus, dass ausgewählte Sachprobleme bestimmte Diskurse evozieren, so z.B. der seit dem Bericht des Club of Rome im Jahre 1972 angestoßene Wandel von Gesellschaften hin zu nachhaltiger Entwicklung, die in einer Reihe von Nachhaltigkeitsdiskursen ihren Niederschlag finden (Grober 2010, Parodi et al. 2010 Banse et al. 2011a, Nielsen et al. 2013,). Das Thema der Nachhaltigkeit, zunehmend bedeutsam in Forschung, Praxis und Öffentlichkeit, bezieht eine Vielfalt von Facetten ein, die aufgrund unterschiedlicher Verständnisse hinsichtlich des begrifflichen Sprachgebrauchs die Kommunikation zwischen Gruppen und Personen erschweren. Die anstehenden und zu kommunizierenden Sachprobleme ziehen sich quer durch die Disziplinen und Branchen und betreffen unterschiedliche bzw. gemischte Gruppen von Experten verschiedener Disziplinen und Nichtexperten aus unterschiedlichen Bildungsschichten. ${ }^{2}$ Entsprechend kommt es zum Mix fachlicher, nicht-fachlicher und alltagssprachlicher Sprachverwendungen. ${ }^{3}$ Dieser komplexe Sachverhalt, der im Rahmen der Terminologielehre und/oder durch fachbezogene Definitionen (Arntz et al. 2004) nicht erfasst werden kann, bildet eine Herausforderung für die Fachkommunikation (vgl. auch Weber 2010).

Als Alternative bietet sich der kognitive Ansatz semantischer Modelle an, die als sozial geprägte Verstehens- bzw. Interpretationsmuster (Schemata) die themen- und situationsbezogene Kommunikation von Wissen prägen (s.u. Abschnitt 2). Wir fokussieren in diesem Beitrag auf ausgewählte Modelle zu Risiko und Resilienz, die zu den zentralen Begriffen der präventiven Krisenbewältigung gehören und die in den verschiedenen Sachdomänen und Kommunikationskontexten unterschiedlich interpretiert werden. Gemeinsam ist ihnen die Nähe zu Krisensituationen und präventiver Krisenbewältigung durch Kommunikation.

Beide Begriffe spielen in den Begriffsfeldern der Nachhaltigkeitsdiskurse eine wichtige Rolle und werden mit Bezug dazu in diesem Beitrag näher beleuchtet. Der Begriff der Nachhaltigkeit wird vorwiegend in der Perspektive der Entwicklung gedeutet (vgl. „Nachhaltige Entwicklung“), die einem zu erwartenden bzw. beobachtbaren Krisengeschehen entgegen $\mathrm{zu}$ setzen sei, das aus einem zunehmend überfordernden Umgang mit Natur und Ressourcen resultiert. Mit den Modellen des Risikos werden die möglichen Gefahren, Gefahrensituationen und Verluste bzw. deren Vermeidung antizipiert, die von außen auf Systeme (im weitesten Sinne) einwirken, mit den Modellen der Resilienz die Stärkung der Widerständigkeit der Systeme selbst, so dass sie diesen Gefahren oder Beschädigungen standhalten bzw. sie durchstehen können. Die jeweiligen Prinzipien für Risiko und Resilienz haben einen hohen Allgemeinheitsgrad und können daher auf verschiedene Domänen angewendet werden, innerhalb derer sie spezifiziert werden.

Die Fragestellung in diesem Beitrag fokussiert auf Kommunikationsprobleme, die auf den unterschiedlichen Verständnisoptionen der zentralen Begriffe beruhen. Unser Zugang erfolgt in (text-)linguistischer Herangehensweise, d.h. auf einer Analyse und

\footnotetext{
${ }^{2}$ Vgl. das Portal zur Vermittlung von Konzepten und Forschungsfragen der Nachhaltigkeit, initiiert durch das BMBF (Bundesministerium für Bildung und Forschung, BRD): www.zukunftsprojekterde.de

${ }^{3}$ Unterschieden werden die disziplinär festgelegten Begrifflichkeiten (Fachsprache im engeren Sinne, z.B. Restrisiko, Risikoformel), die unabhängig von der Einzeldisziplin verwendeten Begriffe (z.B. Organisation, Ressource, Verfahren) und die im Alltag verwendeten allgemeinen Begriffe (z.B. Strom, Obst, mitmachen). Vgl. auch Rothkegel 2012b.
} 
Beschreibung der den Begriffen zugrundeliegenden semantischen Modelle in Abhängigkeit ihres Gebrauchs in Texten. Dabei gehen wir von einem geisteswissenschaftlichen Sprachverständnis aus, nach dem Sprache und Wissen als miteinander verbunden gelten. Diese theoretische Basis wird zunächst im 2. Abschnitt erläutert, bevor wir in den Abschnitten 3 und 4 auf ausgewählte Modelle zu den Begriffen Risiko und Resilienz eingehen. Die Ergebnisse der Analysen fassen wir im 5. Abschnitt mit Blick auf den rahmengebenden Begriff der Nachhaltigkeit zusammen.

\section{SPRACHE, WISSEN UND MODELLE}

Kommunikation wird getragen durch Sprache bzw. andere Zeichensysteme (visuelle, akustische), wodurch Referenz hergestellt wird und Personen kommunikativ aufeinander einwirken können. Dies ist die Basis für Strategien der Verständigung und die Vermittlung von Handlungskompetenz. Gehen wir von einem Sprachverständnis im Sinne von Wilhelm von Humboldt aus, wie es Trabant (2012) nachvollziehbar darlegt, trägt Sprache in zweifacher Weise dazu bei. Einerseits eröffnet sie die kognitive Möglichkeit der begrifflichen Gliederung des Wissens über die Welt (im Sinne von „Weltansichten“), andererseits ermöglicht sie im Sinne von Zeichen deren kommunikative Anwendung. Dabei geht Humboldt von einer prinzipiellen Vielfalt unterschiedlicher Weltansichten aus, die sich in Abhängigkeit von Kulturen und Epochen entwickeln (Trabant, 2012: 90-104). In den Kognitionswissenschaften spricht man von „mentalen Modellen“, also Vorstellungen über die Welt, die als Erklärungs- und Deutungsmuster die Kommunikation von Wissen prägen (ausgehend von Johnson-Laird 1983; u.a. Jacob 1991). Sie haben zu tun mit dem Wissensstand und den subjektiven Einstellungen von Personen, nach denen das „Bild“ von der Realität gefiltert wird (z.B. „unterschiedliche Weltbilder“ in der Biotechnologie-Debatte; vgl. Gill 2012, 65). Modelle beeinflussen nicht nur die Denkweise der Akteure sondern auch deren Handeln und haben dabei den Status von Strategien. Dies gilt gleichermaßen für wissenschaftliche wie für Alltagstheorien und natürlich auch im Hinblick auf komplexe Begriffe wie Risiko und Resilienz.

Die systematische Erfassung von Modellen ist gebunden an den Kontext, in dem die entsprechenden Ausdrücke verwendet werden. Als linguistisch analysierbar gelten demgemäß die Texte, die durch einschlägige Themen und situative Einbindung gekennzeichnet sind (Metzeltin 2007). Insbesondere im Thema und der Themenentfaltung kommen die spezifischen Modelle der jeweiligen Schlüsselbegriffe, hier also Risiko und Resilienz, zum Tragen. ${ }^{4}$ Hinsichtlich weitergehender Analysen ist es wünschenswert, eine einheitliche Beschreibung solcher Modelle verfügbar $\mathrm{zu}$ haben, die auch den systematischen Vergleich gestatten Für die Beschreibung von Modellen bietet sich die Schema-Repräsentation an, wie sie als Wissensrepräsentation in der Form von Bündeln von Attribut-Wertpaaren (anders ausgedrückt als Leerstellen-Füller-Paare oder als Frames) eingeführt sind (Schnotz 1994, Konerding 1993, Ziem 2008). Inhaltlich gesehen handelt es sich um Konfigurationen von allgemeinen Kategorien (Attribute, Leerstellen) und deren Spezifikationen (Werte, Füller), die einen bestimmten Wissensstandard in Bezug auf ein Objekt oder einen Sachverhalt darstellen. Wir skizzieren in diesem Beitrag

\footnotetext{
${ }^{4}$ Zur textlinguistischen Bestimmung von „Thema“ im Sinne von Referenzbereich, Aussage oder Frage vgl. Adamzik 2004: 65. Zur Relation von Modellen und Thema vgl. Rothkegel 2013a.
} 
die Beispiele in Bezug auf derartige allgemeine Kategorien, ohne die formale Darstellung, da hier ohne Relevanz, auszuführen.

\section{RISIKO UND RISIKOKOMMUNIKATION}

\subsection{Zum Begriff Risiko}

Im Alltagsverständnis ist Risiko nur vage erfasst. Gemäß DUDEN (Bedeutungswörterbuch 2002) wird der Ausdruck paraphrasiert mit Wagnis, Möglichkeit des Verlustes oder Misserfolges bei einem Vorhaben (lat. risicare „Klippen umschiffen“). Bei den Zusammensetzungen z.B. Risikooperation) erscheint lediglich Gefahr als Merkmal. Im Rahmen wissenschaftlicher Disziplinen bildet er dagegen eigene Paradigmen der Forschung. Die Risikoforschung, zunächst vornehmlich auf Naturkatstrophen bezogen, dann aber - verstärkt als Reaktion auf eine Reihe technischer Großunfälle in den 80er Jahren - hat sich um eine systematische Sortierung der Begrifflichkeiten bemüht (Banse/Bechmann 1998, Hribal 1999). Diese Arbeiten sind zu sehen innerhalb einer generellen Aufmerksamkeit für die Zunahme von Risiken sowie Anfällig- und Verletzbarkeiten (vulnerability) moderner Gesellschaften durch jegliche Art von Störungen bis hin zu Katastrophen (vgl. Risiko-Gesellschaft: Beck 1986, 2007).

Dabei spielen die disziplinären Zugänge zu Risiko als Thema eine wichtige Rolle. So stehen Aspekte der Kontrollierbarkeit bzw. Unkontrollierbarkeit im Vordergrund naturwissenschaftlich-technischer Perspektiven. Im Bereich von Wirtschaft und Finanzen geht es um Gewinn (Wagnis) und Verlust (Schaden). Im Bereich der Sozialwissenschaften kommen die beteiligten Menschen als Akteure und Betroffene in den Blick. In interdisziplinären Diskursen (z.B. Nachhaltigkeitsdiskurse) kommt es zu gemischten Perspektiven. Dennoch bleibt der Begriff des Risikos theoretischer Art, der in seiner Zukunftsorientierung nur in der Kommunikation existiert und hier gebildet, spezifiziert und verändert wird. So gehört eine Reihe weiterer Begriffe zum elaborierten Begriffsfeld der Arbeit an Risikoaspekten, u.a. Risikowahrnehmung, Risikoanalyse, Risikoidentifikation, Risikoeinschätzung, Risikobewertung.

\subsection{Risiko und Krise}

Als übergeordnet für die Gesamtstrukturierung eines Risikos gilt ein Ereignis (Schadensereignis, Unfall, Anschlag, Katastrophe). Semantisch gesehen handelt es sich um die Relation von zwei Zuständen, wobei das Ereignis einen Ausgangszustand in einen Ergebniszustand überführt. Diese Überführung erfolgt über ein Geschehen (Prozesse, Vorgänge) oder über Handlungen (von Akteuren intendierte bzw. nicht-intendierte Einwirkungen). Der Ergebniszustand kann nun aus verschiedenen Gründen erwünscht sein oder unerwünscht. Situationen, von denen aus es zu unerwünschten Ergebnissen kommen kann, werden als Risikosituationen bezeichnet (ausführliche Darstellung in Rothkegel 2010b). Ein besonderer Stellenwert kommt den Kipp-Situationen zu. Geht man davon aus, dass ein Ereignis durch eine Kette von Teilereignissen zustande kommt, können in der Fortsetzung solcher Ketten spezifische Zustände entstehen, die beide Möglichkeiten für eine Weile offen halten, d.h. es gibt Situationen, von denen aus sich das Geschehen in die eine oder andere Richtung entwickelt (alltagssprachlich und metaphorisch: auf Messers Schneide, auf der Kippe stehen, sich zum Guten/Schlechten wenden). Ziehen sich solche Situationen in die Länge oder gibt es ein mehrfaches Hin und 
Her zwischen beiden Entwicklungsrichtungen, spricht man von einer Krise (Finanzkrise, Klimakrise, Gesundheitskrise).

Krisen verlangen nach Interventionen (Gegenmaßnahmen), die jeweils als Reaktionen auf spezifische Aspekte unerwünschter Situationen geeignet sind. Mit Bezug auf die solche Situationen herbeiführenden Ereignisse ergibt sich eine Einteilung in Abhängigkeit der zeitlichen Dimension des jeweiligen Ereignisses: davor (Vor-Ereignis: Prävention), während (Ereignisablauf: Warnung, Rettung), danach (Nach-Ereignis: Rekonstruktion). Im Hinblick auf die Krise hat der Begriff des Risikos mit allen drei Dimensionen zu tun, darüber hinaus umfasst er gleichzeitig die Dimension der erwünschten Situationen. Ersteres ist gekennzeichnet durch die drei Perspektiven: Gefahr und Schaden (Ereignisbezug) sowie Sicherheit (Maßnahmen-Bezug), Letzteres durch die Perspektive des Wagnisses, die den Anteil von Gewinn und Vorteil kennzeichnet. Im Folgenden skizzieren wir die vier genannten Risikomodelle in den Perspektiven Gefahr, Schaden, Sicherheit, Wagnis (vgl. Rothkegel 2008; multilinguale Perspektive in Rothkegel 2012a).

\subsection{Risikomodelle}

(a) Risiko in der Perspektive Gefahr: Gefahren bzw. Gefährdungen/Bedrohungen stehen im Fokus. Das Ereignis selbst ist im Hinblick auf sein Gefährdungs- oder Bedrohungspotenzial spezifiziert. Das Spektrum der Möglichkeiten ist groß und vielfältig. Es reicht von der Nutzung einfacher technischer Produkte im Alltag, über Großtechnologien oder natürliche Gefährdungen (Hochwasser, Erdbeben, Tsunami, Vulkanausbrüche) bis $\mathrm{zu}$ groß angelegten intendierten Attacken (z.B. terroristische Anschläge). Die Kommunikation selbst bildet einen Bestandteil der Maßnahmen, wofür es rechtliche und dokumentarische Standards gibt (soweit sie nicht der politischmilitärischen Geheimhaltung unterliegen). Die Themenentfaltung orientiert sich an den potenziellen Ereignissen, zumeist in einer Art Negativform. Konstruiert werden Szenarien dazu „was nicht sein soll“, um diese in der Realität zu verhindern (Prävention). In der Antizipation dessen, „was sich trotzdem ereignen kann“, geht es um Maßnahmen des Schutzes (Vorsorge und Rettung). Geschieht die Nutzung im Interesse der Nutzer, kann man von der Akzeptanz der Maßnahmen ausgehen. Im Hinblick auf Abwehr oder Schutz vor Attacken ist mit Konflikten zu rechen (Beschränkung von Zugangsberechtigungen, Einschränkung von individuellen Rechten, z.B. Privatsphäre). Im Hinblick auf die Interaktionen stehen Handlungen des Informierens, Instruierens und Warnens im Vordergrund.

Der Blick auf die Akteure impliziert im Weiteren Unterscheidungen von Gefahren nach deren Verursachung wie nature-made und men-made mit fließenden Übergängen, wobei in der öffentlichen Debatte auch Naturkatastrophen als men-made eingeschätzt werden (vgl. Klimadebatte). Aber auch im technischen Bereich verschiebt sich die Aufmerksamkeit vom technischen Produkt auf die Handlungen der Akteure beim Umgang mit Technik. In diesem Sinne führt der Technikphilosoph Ropohl (2009) den Begriff des „Soziotechnischen Systems“ ein, in dem „menschliche und sachtechnische Subsysteme eine integrale Einheit bilden“ (Ropohl 2009, 141).

Was bedeutet nun Gefahr im konkreten Sinne? Wichtig ist die Gefahrenquelle, die im negativen Sinne auf eine andere Einheit einwirkt. Für diese stellt sie insofern eine Bedrohung (engl. hazard) dar, als die betroffene Einheit zu Schaden kommen kann, d.h. Opfer werden kann (z.B. Elektrizität als Art der Bedrohung und Stromschlag als Schaden). Betrachtet man die Techniknutzung als Interaktion zwischen Akteur, 
technischem System und Umwelt kommen alle drei gleichermaßen als Gefahrenquelle wie auch als Opfer in Frage. Akteure können Fehler machen (in der Konstruktion oder bei der Bedienung mit „menschlichem Versagen“) oder als „böse“ Angreifer Technik für eine Schädigung anderer missbrauchen (Attacke, Anschlag, Sabotage). Technische Systeme bilden Gefahrenquellen auf verschiedenen Ebenen. Dabei ist grundsätzlich das systeminterne Gefahrenpotenzial $\mathrm{zu}$ beachten (z.B. Elektrizität, Gefahrenstoffe, Bewegungen von Robotern, Kernschmelze). Aber auch Defekte (durch Transport oder Gebrauch) können zur Gefahrenquelle werden (z.B. beim Kühlgerät Austreten von Kühlmittel aufgrund einer Beschädigung des Behälters) oder das Versagen von Kontrollmechanismen (,technisches Versagen“). Einwirkungen aus der Umwelt wiederum, denen in der Konstruktion oder mit der Einschränkung von Gebrauchsweisen Rechnung getragen wird, können sich der Kontrolle teilweise oder gänzlich entziehen (z.B. Wetterverhältnisse bei der Nutzung von Verkehrsmitteln). Auf der Grundlage solcher Basiskomponenten ergeben sich eine Reihe denkbarer Konfigurationen, die als Gefahrensituationen bzw. konkret als Gefährdungsszenarien erfasst werden können.

(b) Risiko in der Perspektive Schaden: Schäden, Verluste, Kosten gehören zu den tragenden Merkmalen naturwissenschaftlich-technischer Modelle. Als attraktiv gilt das Verständnis vom Risiko als Kalkül und der darauf aufbauenden Kontrollierbarkeit von Vorgängen. Im Zentrum steht die Risiko-Formel mit der Berechenbarkeit des Risikos durch die Relationierung von Eintrittswahrscheinlichkeit eines möglichen Schadensereignisses und dem Schadensausmaß. So lässt sich z.B. der Infektion durch eine Grippe im Herbst eine relativ hohe Wahrscheinlichkeit des Eintretens bei relativ geringem Schaden zuordnen, während ein KKW-Unfall relativ selten ist, aber einen extrem hohen Schaden zur Folge haben kann.

Es gibt Graduierungen des Risikos wie „akzeptabel / nicht-akzeptabel“, „tolerierbar / nicht-tolerierbar“. Die jeweiligen Zuordnungen sind abhängig von den beteiligten Individuen, Gruppen, Organisationen. Dabei werden, soziologisch gesehen, Akteure im Sinne von Risikoproduzenten und Entscheidern sowie Betroffene unterschieden. Dazu kommen weitere Professionelle (Wissenschaftler, Behörden), die theoretisch und/oder praktisch mit der Risiko-Bewältigung zu tun haben. Einen hohen Stellenwert haben dabei die Risiko-Wahrnehmung als Voraussetzung, des Weiteren Risiko-Analyse, RisikoIdentifikation, Risiko-Bewertung als Grundlage für die Maßnahmen (s.u. Perspektive Sicherheit).

(c) Risiko in der Perspektive Sicherheit: Sicherheit wird fachlich verstanden im Sinne von „Umgang mit Gefahren““. Hier spielen Kategorien wie Kontrollierbarkeit bzw. NichtKontrollierbarkeit eine zentrale Rolle. Es geht es um konkrete, festgelegte Maßnahmen (Kontrolle) im Umgang mit definierten Gefahren. Diese Maßnahmen bilden eine Kombination der beiden vorgenannten Modelle mit den Kernaspekten Gefahr und Schaden. Im Zentrum stehen die Abwehr von Gefahren und der Schutz der Geschädigten (Schadensobjekte).

Diese wie die grobe Zuordnung der Maßnahmen von Abwehr und Schutz kennzeichnet die Sicherheitsforschung, die die Risikoforschung abgelöst hat, wie auch die Praxis der letzten Jahre. Einen Überblick zur Forschungslage vermittelt der von der Deutschen Akademie der Technikwissenschaften (acatech) herausgegebene Sammelband

\footnotetext{
${ }^{5}$ Vgl. die Alltagsbedeutung von Sicherheit im Sinne von „ohne vorhandene Gefahr“ (Ausführung in Rothkegel 2011b).
} 
„Sicherheitsforschung - Chancen und Perspektiven“ (hg. von Winzer/Schnieder/Bach 2010; vgl. Überblick über nationale und internationale Forschungsprogramme in Thoma/Drees/Leismann 2010: 14-27). Für die Entwicklung unseres Sicherheitsmodells „Abwehr und Schutz“ beziehen wir uns vorwiegend auf die dort zur Diskussion gestellten Beiträge, im Weiteren auf Stieniczka 2006 und Giebel 2012. Eine Brücke zwischen den beiden Kernbegriffen Risiko und Gefahr ergibt sich, wenn Sicherheit verstanden wird als „Abwesenheit eines unvertretbaren Risikos und Gefahr als Anwesenheit eines unvertretbaren Risikos“ (vgl. Regenfuß/Viehweg, 2010: 132 in Anlehnung an DIN Fachbericht 144 zur Sicherheit).

Die jeweils entwickelten bzw. zu entwickelnden Maßnahmen operieren in zwei Richtungen. Einerseits geht es um Abwehrmaßnahmen hinsichtlich der Gefahren, andererseits um Schutzmaßnahmen hinsichtlich der Betroffenen bzw. geschädigten Objekte, Umwelt, Infrastrukturen usw. Abwehrmaßnahmen orientieren sich an den Gefahrenquellen. Wichtig sind Vermeidung, Verhinderung oder zumindest Verringerung von Schaden. Tendenziell und vom Konzept her geht man davon aus, dass die Gefahr kontrolliert und Schaden vermieden werden kann. Bei den Schutzmaßnahmen geht man dagegen davon aus, dass nicht alle Gefahren abgewendet werden können und dass man mit Schadensereignissen realistischerweise rechnen muss. Dabei sollen die Betroffenen und vor allem die Einsatz- bzw. Rettungskräfte aber soweit möglich vor den Folgen geschützt werden (z.B. durch Schutzanzüge bei Bränden oder KKW-Störfällen).

Der Gegenüberstellung von Abwehr und Schutz entspricht die Gegenüberstellung von aktiver und passiver Sicherheit. Im Kfz-Bereich spricht man von aktiver Sicherheit, wenn die Vorrichtungen in spezifischer Weise auf bestimmte Gefahren hin konstruiert sind, so etwa Bremssysteme oder Fahrspur-Assistenten. Passive Sicherheit hat dagegen mit der Schadensbegrenzung bei einem Unfall zu tun, so etwa die Verwendung von Gurten (rechtlich unterstützt durch Anschnallpflicht) oder von Airbags. Stieniczka (2006) verfolgt auf eindrucksvoll belegte Weise, wie sich das vormalig vorherrschende Verständnis von aktiver Sicherheit (,es kann nichts passieren“) langsam zu einem Denken in Kategorien passiver Sicherheit (,es kann was passieren, aber es gibt einen Schutz“) wandelte.

(d) Risiko in der Perspektive Wagnis: Die Gewinnaussicht überwiegt die Angst vor Verlusten (z.B. Wirtschaft, Finanzen, Wetten, Extremsport) bzw. die Kosten sind gegenüber dem $\mathrm{zu}$ erwartenden Gewinn nur gering. Unter ökonomischen Gesichtspunkten, aber auch im Alltag, verbinden sich mit dem Begriff des Risikos bestimmte Vorstellungen von Situationen, in denen Ereignisse oder Handlungen vor allem einen großen Vorteil (Nutzen) und einen geringen Nachteil (Schaden) bringen. Man geht das Risiko eines Schadens ein (Risikobereitschaft), wenn man gleichzeitig an einen Vorteil und das Nicht-Eintreffen des Schadens glauben kann oder wenn der Nutzen den erwarteten Schaden überwiegt. Es stellen sich Fragen danach, wem etwas nutzen oder schaden kann. Auch geht es um Relevanz, Notwendigkeit und die Betrachtung möglicher Alternativen. Bekannt sind entsprechende Debatten und Kontroversen zwischen Risikoproduzenten und Risikobetroffenen.

In dem von acatech initiierten und herausgegebenen Sammelband $\mathrm{zu}$ den Kontroversen um die Biotechnologie (Weitze/Pühler 2012) wird mehrfach betont, dass der mögliche oder versprochene medizinische Vorteil/Nutzen (wissenschaftlicher Fortschritt) die Akzeptanz für die Rote Biotechnologie (Medizinbereich) bei den Nichtexperten begünstigt, während man hinsichtlich der Grünen Biotechnologie (Agrarbereich) tendenziell keine Akzeptanz aufbringt, weil, so die Argumentation (der 
Bauern als Nutzungsexperten), diese unnötig sei und keinen Nutzen bringt (zu den unterschiedlichen Ausgangssituationen in den USA und Europa vgl. Bonfadelli 2012, 217-218). Hier zeigt sich deutlich, dass Risikowahrnehmung und Risikoeinschätzung stark von den lokalen Gegebenheiten wie den Interessen der jeweiligen Gruppierungen abhängen. Entsprechend gelten wissenschaftlicher Fortschritt und wirtschaftliche Vorteile der Biotechnologie insgesamt als Nutzen insbesondere bei den Gruppen, die zu den Risikoproduzenten gehören und für die sich das „Wagnis“ (der einzusetzenden Kosten) offensichtlich rechnet.

Aufgrund der prinzipiellen Zukunftsorientierung und Offenheit von Prognosen erscheinen die Versprechen der Risikoproduzenten zwangsläufig mit deren Glaubwürdigkeit gekoppelt. Ohne allseitig begründete Vertrauensbasis kann in diesem Kontext keine ,gedeihliche“ bzw. „gelingende“ Kommunikation stattfinden. In diesem Sinne ist die Kommunikationskrise im Hinblick auf das Thema Grüne Biotechnologie eher als Vertrauenskrise zu verstehen, denn durch Mangel an Information begründet.

\section{RESILIENZ}

\subsection{Zum Begriff der Resilienz}

Der Begriff der Resilienz (lat. resilire: abprallen, zurückspringen), im Deutschen weniger gebräuchlich, wandert in den letzten Jahren durch die verschiedenen Disziplinen und wird entsprechend differenziert paraphrasiert. In der Physik bzw. Materialwissenschaft spricht man von Elastizität, in der Medizin, wo das Konzept seinen Ursprung haben soll, von Widerstandsfähigkeit, in der Psychologie vom Vermögen, Lebenskrisen ohne anhaltende Beeinträchtigungen durchzustehen (vgl. Giebel 2012: 90). In der Grundbedeutung ist Resilienz demnach generell zu verstehen als eine Fähigkeit von Systemen sowie deren Verfügen über Ressourcen für adäquate Reaktionen auf Unsicherheiten. Dazu gehören, zitiert nach Giebel (2012: 94): "Prävention (to prevent something bad from happening), Proaktion (to prevent something bad from becoming worse), Reaktion (to recover from something bad once it has happened)".

Eine andere Prägung charakterisiert die Kommunikation, wenn es hervorgehobene Eigenschaften und Fähigkeiten eines Systems sind, die die Thematik der Krisenbewältigung bestimmen statt der es bedrohenden Gefahren, Verluste und Schäden, wobei das unerwünschte Ereignis eher in den Hintergrund tritt und stattdessen Aspekte von Resilienz im Sinne von aktiver Widerständigkeit, Regenerationsfähigkeit, Einfallsreichtum sowie Fähigkeiten zur Identifikation von Ressourcen und Handlungsalternativen, zu Kooperation und Vertrauen in den Vordergrund rücken. Die Spezifikationen werden erst in der Kommunikation entwickelt, so wie die Fähigkeit zur Kommunikation wiederum die Resilienz eines Systems erhöht (vgl. www.ssu.ethz.ch/Aktuell/CSS-Analysen).

Generell geht man davon aus, dass in komplexen Umfeldern mit Irritationen zu rechnen ist, die nicht ohne weiteres antizipiert werden können, so dass Modelle der gezielten Abwehr und des Schutzes im Sinne von Sicherheitsmaßnahmen nicht greifen und Risikodebatten in ihrer beschränkten Dimension nicht ausreichen. Stattdessen sind integrierte Ansätze gefragt, die Eigenschaften sowie Verhaltens- und Handlungsweisen von Systemen in ihrer Dynamik und Wechselwirkung mit anderen Systemen erfassen.

Als Fachausdruck gehört der Begriff der Resilienz mehreren Disziplinen (u.a. Psychologie, Soziologie, Pädagogik, Medizin, Physik, Informatik) und interdisziplinären 
Forschungsrichtungen an (u.a. Risiko- und Sicherheitsforschung, Gesundheitsforschung), die seine Bedeutung in unterschiedlicher Weise akzentuieren. Übergreifend bezieht er sich auf Fähigkeiten von Systemen, Krisen standzuhalten, eigene Schäden zu minimieren, schnell zu regenerieren und beschädigte bzw. verlorene Funktionsfähigkeit zurück zu erlangen. Von Interesse sind vor allem natürliche (einschließlich biologische), soziale, ökonomische oder technische Systeme. Objekte, Umwelt, Infrastrukturen sowie Gruppen und Gesellschaften, aber auch Individuen stehen im Fokus. Relevant ist, dass in allen Fällen die Kommunikation eine wichtige Rolle spielt. Sie bildet den Raum, in dem Bedeutung hergestellt und vermittelt wird.

Die beteiligten Begriffsfelder sind naturgemäß komplex und unterscheiden u.a. Robustheit mit Kategorien wie Anfälligkeit, Empfindlichkeit, Vulnerabilität; Widerstandsfähigkeit, Redundanzen und Resilienz mit Kategorien wie Widerständigkeit, schnelle Regenerations- und Reaktionsfähigkeit, Ressourcenkompetenz, Einfallsreichtum, Kommunikationsfähigkeit, Kooperation. Wir skizzieren im Folgenden zwei Modelle der Resilienz, zum einen im Rahmen der Gesundheitskommunikation, zum anderen mit Blick auf resiliente Gesellschaften.

\subsection{Resilienz und Gesundheit}

Im Gesundheitsbereich entspricht die Unterscheidung von erwünschtem bzw. unerwünschtem Zustand den Polen von Gesundheit und Krankheit. Beide sind miteinander verbunden in Handlungen der Bewältigung (Überführung) unerwünschter Zustände in Richtung erwünschter Zustände. Resilienz deckt dabei den Bereich der Handlungen $\mathrm{ab}$, der sich auf Prävention (Vermeiden unerwünschter Zustände) und Schadensverringerung bei nicht möglicher Prävention bezieht.

Das Thema Gesundheit prägt eine Vielzahl von Kommunikationsgemeinschaften, die sich sowohl in der personalen Zusammensetzung, in Zielen und Interessen, in Intensität und Dauer der Interaktion unterscheiden (z.B. Fachgemeinschaften, öffentlicher Diskurs, Bereiche wie Diagnose und Therapie oder Forschung und Beratung). Unterstützt durch Web 2.0-Technologien erweitert sich der Kreis der an der Kommunikation Beteiligten. Wünsche nach Partizipation mit zunehmendem Interesse am Wissensaustausch führen dazu, dass sich die Rollen von Experten und Nichtexperten in der Kommunikation untereinander vermischen. Konkurrierende Schulen innerhalb der medizinischen Disziplinen auf der einen Seite, unterschiedliche Facetten des Erfahrungswissens der (potenziellen) Patienten auf der anderen Seite treffen in der Kommunikation aufeinander: bei der Nutzung der Medien (Wikipedia), im persönlichen Arzt-Patient-Gespräch, bei der Beratung von Kunden durch die Apotheken (z.B. Kundenzeitschriften, Portale) oder in Interaktionen der Patienten untereinander in organisierten Selbsthilfegruppen sowie in freien Interaktionen im Social Web. In allen Situationen kommt es zum Mix von Fachund Alltagswissen, ausgedrückt im Mix des Gebrauchs von Fach- und Alltagssprache mit den bekannten Konsequenzen. Neben gelungener Kommunikation gibt es Missverständnisse, Konflikte, Nicht-Akzeptanz und gescheiterte Therapien.

Je nach Modell von Gesundheit und Krankheit kommen unterschiedliche Diagnosen und Therapievorschläge zum Tragen, deren Systematik für das Verständnis der Patienten und damit für deren Akzeptanz bei der Therapie von hoher Relevanz ist. Umgekehrt bringen Gesundheitssuchende ihre geltenden Vorstellungen mit den Berichten über Zustände oder Beschwerden ein. Einen Überblick über die Vielfalt von Modellen für Gesundheit und Krankheit gibt Franke (2010), wobei Gesundheit als in geringerem 
Umfang als die verbreiteten Modelle vom Kranksein und Krankheit beachtet erscheint. Als Verständnistypen kommt sie auf sechs jeweils auf das Individuum bezogene Interpretationsmuster: Gesundheit als Störungsfreiheit, Wohlbefinden, Leistungsfähigkeit und Rollenerfüllung, Gleichgewichtszustand, Flexibilität und Anpassung. Die WHO 1986 zählt eine Reihe von darüber hinausgehenden Bedingungen auf, die sich an Gesellschaften orientieren und in einem globalen Rahmen weitere Aspekte wie solche der Nachhaltigkeit einbeziehen: Frieden, angemessene Wohnbedingungen, Bildung, Ernährung, Einkommen, ein stabiles Ökosystem, eine sorgfältige Verwendung vorhandener Naturressourcen, soziale Gerechtigkeit und Chancengleichheit (www.who.int/healthpromotion/conferences/previous/Ottawa).

Begriffsfelder zur Resilienz, die im Bereich der medizinisch-psychologischen Gesundheitskommunikation relevant sind, sind einerseits gekennzeichnet durch die Betonung der Ressourcenkompetenz und ausgewählte Gesundheitsmodelle, während der Bereich der Risikofaktoren, die ebenfalls eine Rolle spielen, eher an Krankheitsmodellen orientiert ist. Mit Ressourcenkompetenz sind Kontexte (Situationen, Szenarien) angesprochen, in denen Konzepte wie Gesundheitsförderung, Stärkung von Ressourcen, Selbstbestimmung und Selbstwirksamkeit ihren Platz haben. Begriffsfelder mit Bezug zu den Risikofaktoren sind gekennzeichnet durch Kontexte von Gefahr, Schaden und Sicherheit (vgl. Abschnitt 3.3).

Mit der Nähe zum Begriff der Salutogenese (Gesundheitsentstehung; vgl. salus = lat. Wohlbefinden) gehört der Resilienzbegriff zu Feldern, die als Alternative zu denen der Pathogenese (Krankheitsentstehung) verstanden werden (Fabry 2011). Damit kommen zwei komplementäre Perspektiven auf Gesundheit bzw. Krankheit in den Blick. In der pathologischen Ausrichtung stehen die Entstehungsbedingungen für Krankheiten als zentrale Forschungsthemen im Vordergrund, eindeutige Ursache-Wirkungsbeziehungen bieten die Orientierung. Ausgangspunkt ist hier ein Defizit-Modell, in dem der Begriff der Vulnerabilität (Verletzbarkeit) eine wichtige Rolle spielt. Grundsätzlich steht die Vulnerabilität von Personen oder sozialen Systemen in Relation zu äußeren ungünstigen Einflussfaktoren. Ziel ist hier die Minimierung der Folgen (Schadensbegrenzung). Bei der salutogenetischen Denkweise geht es dagegen um die Mobilisierung von Widerstandsreserven. In dieser Sicht bildet der Begriff der Resilienz (als erlernbare Kompetenz) das entsprechende Gegenstück zur Vulnerabilität. Gesundheit wird dagegen eher in einer vernetzten Betrachtungsweise erfasst, in der biologische, psychologische und soziale Faktoren zusammenwirken. In diesem Sinne bilden Gesundheit und Krankheit statt getrennte Zustände die Pole eines Kontinuums, in dem sich der Patient positioniert. Aus ärztlicher Sicht wird in der Folge den Bewältigungsressourcen besondere Aufmerksamkeit geschenkt (Fabry 2011).

In den 70er Jahren wurde das Konzept der Salutogenese durch den Medizinsoziologen Aaron Antonovsky (Antonovsky 1979, Antonovsky/Franke 1997, Schiffer 2001) entwickelt, das mehr und mehr auch gegenwärtig Forschungsansätze prägt. Drei Dimensionen gelten als konstitutiv, die unter dem Begriff der Kohärenz bzw. des Kohärenzsinnes zusammengefasst werden: 1. Gefühl der Verstehbarkeit als kognitive Komponente im Sinne einer Regelmäßigkeit der Dinge bzw. Fähigkeit, Reize als geordnete konsistente, strukturierte Information verarbeiten $\mathrm{zu}$ können (comprehensibility), 2. Gefühl der Handhabbarkeit oder Bewältigbarkeit im Sinne eines Gleichgewichts zwischen Einsatz und Ergebnis, als Überzeugung, dass Schwierigkeiten lösbar sind und dass man über die dafür geeigneten Ressourcen verfügt (manageability) 
und 3. Sinnhaftigkeit oder Bedeutsamkeit im Sinne einer emotionalen Komponente und man das Leben als emotional sinnvoll empfindet, „dass wenigstens einige vom Leben gestellte Probleme und Anforderungen es wert sind, dass man Energie in sie investiert, dass man sich für sie einsetzt und sich ihnen verpflichtet, dass sie eher willkommene Herausforderungen sind als Lasten, die man gerne los wäre (meaningfulness) (Antonovsky/Franke 1997). Das Programm zur Förderung der Widerstandskraft war zunächst mit Bezug auf Kinder mit schwerer Kindheit erforscht worden, die als Erwachsene dennoch in der Lage waren, ein erfolgreiches Leben zu führen. Heute stehen psychoterapeutische und pädagogische Programme im Vordergrund, die auf ähnlichen Konzepten basieren und die sich mit dem Schutz von Kindern und Jugendlichen vor Drogen und Kriminalität beschäftigen (Hermann 2009). Dabei stehen Kategorien im Vordergrund wie Belastbarkeit, Elastizität, Widerstandsfähigkeit.

Eine weitere Schiene bezieht sich seit den 90er Jahren auf Erwachsene und Risikofaktoren durch die Belastungen der Arbeitswelt (vgl. Coaching-Programme, Siegrist 2009). Interessant in diesem Zusammenhang ist eine Interviewstudie zum Thema „Resilienz im Arztberuf“, die als Projekt 2010/2011 am Universitätsklinikum Heidelberg durchgeführt worden ist, vgl. Zwack 2013). Motiviert ist dieses Projekt durch die Annahme, dass der Arztberuf zu den besonders gesundheitsgefährdenden Tätigkeiten gehört. Zu den Ergebnissen gehört eine Liste möglicher Resilienz-Kategorien wie:

- $\quad$ proaktiv-offene Kommunikation gegenüber Kollegen

- proaktiv-offene Kommunikation gegenüber Patienten

- $\quad$ Abgrenzung/Selbstschutz gegenüber Patienten

- Ziel- und Standortbestimmung

- $\quad$ Pflege der Professionalität

- $\quad$ Selbstorganisation

- Fehlermanagement

- $\quad$ Einzelsupervision

- Grundhaltungen

- Akzeptanz und Realismus

○ Selbstbewusstsein und Reflexivität

- Handlungsorientierung

- Innere Distanzierung

Schließlich steht Resilienz für „Wachsen in und durch Krisen“ und wird als Chance begriffen, neue Kompetenzen zu entdecken und zu entwickeln (Walsh 2003). Generell gilt Resilienz als ein zukunftsorientierter Prozess mit Entwicklungsperspektive, wobei Wissen über Schutz- und Risikofaktoren in der Praxis für Prävention und Therapie eingesetzt werden können (Hermann 2009).

\subsection{Resiliente Gesellschaften}

Seit dem Hurrikan Katrina in den USA im Jahre 2005 wird das Konzept der Resilienz verstärkt auf gesellschaftliche Dimensionen bezogen. Forschungen betreiben u. a. die ETHZ (Zürich, Center for Security Studies, CSS) ${ }^{6}$ sowie die Universität Stockholm mit ihrem Stockholm Resilience Centre ${ }^{7}$, das auf sozial-ökologische Resilienz spezialisiert ist. Trachsler (2009) skizziert in der vom CSS herausgegeben Zeitschrift CSS Analysen zur

\footnotetext{
${ }^{6}$ www.ssn.ethz.ch

${ }^{7}$ www.stockholmresilience.org
} 
Sicherheitspolitik die Charakteristika für den Aufbau hoch resilienter Gesellschaften. Es sind vier Punkte, die den beiden Bereichen Schadensbegrenzung/ Bereitschaft und Krisenreaktion/Erholung zugeordnet werden. $\mathrm{Zu}$ ersterem Bereich gehören die Eigenschaften Robustheit (robustness) und Redundanz (redundancy), zu letzterem die Fähigkeiten Einfallreichtum (resourcefulness) und Schnelligkeit (rapidity). Ein (technisches oder soziales) System gilt als robust, wenn es Belastungen standhält. Mit Redundanz ist gemeint, dass alternative Möglichkeiten zur Erfüllung kritischer Aufgaben zur Verfügung stehen. Einfallsreichtum entspricht der Kapazität zur kreativen und angemessenen Reaktion auf ein Schadenereignis. Schnelligkeit kennzeichnet eine rasche Reaktions- und Regenerationsfähigkeit. Als grundlegend gilt, dass Konsens über relevante Herausforderungen besteht, dass die Risiken verteilt sind und die Reaktionen auf das Eintreten eines Ereignisses koordiniert werden.

Von besonderem Interesse ist, dass der Kommunikation ein hoher Stellenwert eingeräumt wird. Kommunikation ist wichtig für Vorsorge und Planung, für die Verbindung von Öffentlichkeit und privaten Ebenen, für den Informationsaustausch und die Bildung von Partnerschaften und Netzwerken. Als Barriere für die Umsetzung einer Resilienz-Politik gilt letztlich der Mangel an einem gemeinsamen Verständnis des Konzepts der Resilienz (Trachsler 2009: 3).

\section{RISIKO UND RESILIENZ IM RAHMEN DER NACHHALTIGKEIT}

Die Kommunikation über Nachhaltigkeit ist facettenreich und unübersichtlich (Fischer/Hahn 2001). Wir beziehen uns im Folgenden auf eine linguistische Perspektive, wie sie in Rothkegel (2013b) dargelegt ist. Dabei geht es um einen komplexen Schlüsselbegriff, der eine Reihe weiterer Begriffsfelder einbezieht, u.a. Generationengerechtigkeit, soziale Gerechtigkeit, Gesundheit, Biodiversität, Klimawandel, Partizipation, Regenerierbarkeit, Ressourcenschonung, Umweltschutz, Sicherheit, Resilienz, Effektivität, Effizienz und Suffizienz im Sinne von Genügsamkeit. Grober (2010: 14) umreißt den Begriff wie folgt: „... Nachhaltigkeit [ist] der Gegenbegriff zu „Kollaps“. Er bezeichnet, was standhält, was tragfähig ist, was auf Dauer angelegt ist, was resilient ist und das heißt: gegen ökologischen, ökonomischen und sozialen Zusammenbruch gefeit." Insgesamt zielt der Nachhaltigkeitsdiskurs auf ein alle Lebensbereiche umfassendes Handeln im Sinne eines aktiven Bewahrens von Bewahrenswertem bei gleichzeitiger Zukunftsorientierung und der Entwicklung von Potenzialen. Dies geht weit über den aus der Forstwirtschaft des 18. Jahrhunderts stammenden Grundgedanken, die Bewirtschaftung von natürlichen Ressourcen mit Blick auf ihre Regenerationsfähigkeit zu beschränken (zur Geschichte des Begriffs vgl. Grober 2010). Als Leitbegriff für die Arbeit der Weltkommissionen (z.B. von Rio 1992 bis Rio 2012) unterliegt er einer kontinuierlichen Veränderung im Hinblick auf eine stärkere Konkretisierung und Operationalisierung.

Der gegenwärtige Nachhaltigkeitsdiskurs ist geprägt durch das so genannte DreiSäulen-Modell, das an den Disziplinen Ökologie, Ökonomie und Soziales ausgerichtet ist. In diesem Sinne repräsentiert das vom BMBF initiierte Portal zur öffentlichen Vermittlung von Inhalten und Forschungsfragen das Ineinandergreifen der relevanten Themen (www.zuknftsprojekt-erde.de). Im Weiteren wird ein Vier-Säulen-Modell diskutiert, wobei Kultur als vierte, die anderen verbindende Säule in den Vordergrund gestellt wird (Parodi et al. 2010, Banse et al. 2011a). Der Kulturbegriff wird in dieser 
Debatte in einer erweiterten Fassung verstanden. So beziehen sich Hauser/Banse (2010) in genereller Weise auf Herder und dessen wertneutrales Kulturverständnis im Sinne „spezifischer Lebensweisen einzelner Kollektive“ (Hauser/Banse 2010: 23) und in spezieller Weise auf den Kulturwissenschaftler Hansen (Hansen 2004/2010). Nach Kopfmüller (2010: 43) sind betroffen ,,alle Prozesse des Umgangs der Menschen mit sich und der natürlichen Umwelt. Dabei einbezogen sind Rechts-, Wirtschafts-, Sozial- und Politikordnungen“. Mit einem solchen weiten Konzept von Kultur ist es möglich, die häufig kommunizierten Verengungen der Nachhaltigkeitsdebatte auf Umweltaspekte bzw. auf herkömmliche technische Problemlösungen mit dem vorherrschenden EffizienzLeitbild zu verlassen. An die Stelle treten andere Prinzipien (z.B. Effektivität, Suffizienz, Qualität statt Quantität, Langlebigkeit von Produkten, Reparatur und Recycling statt Wegwerfen, Müllvermeidung) sowie die Veralltäglichung von Nachhaltigkeit mit der Einbeziehung des Individuums (vgl. Hübner 2010, 2011). In dieser Sicht erhalten die oben skizzierten Modelle zu Risiko und Resilienz einen gewichtigen Stellenwert. Sie sind Bestandteile einer ganzheitlichen Umgestaltung der Produktions- und Konsumtionsketten. Dabei zielen sie eben nicht auf eine schöne, heile, nachhaltige Welt, sondern auf Situationen mit Konflikten, Krisen und Kontroversen.

Risiko wie Resilienz sind grundsätzlich auf Zukunft ausgerichtet, was sich mit der Grundlinie des Konzepts der nachhaltigen Entwicklung deckt. Die Beschäftigung mit Risiken weitet den Blick für die Voraussetzungen des Gelingens. Resilienz im Sinne des Standhaltens und Überstehens bezieht sich auf den Kerngedanken der Nachhaltigkeit. Der Modell-Ansatz liefert eine Struktur für Operationalisierung im regionalen Bezug wie auch für Generalisierung im globalen Bezug. Als Beispiel können Gesundheitsmodelle (im Sinne der Salutogenese) gelten, die individuelle bis globale Einzugsbereiche aufweisen. So reicht „Gesundheit" vom Wohlbefinden des Individuums bis hin zu sozialer Gerechtigkeit als politischer Zustand einer Gesellschaft. Resilienz bezieht den Wirkbereich vom einzelnen Individuum bis zu komplexen Gesellschaften ein.

Der Beitrag hat versucht, anhand ausgewählter Modelle zu Risiko und Resilienz einige grundlegende Linien durch das begriffliche Terrain in Nachhaltigkeitsdiskursen zu ziehen. Er hat zudem deutlich gemacht, dass die systematische Klärung semantischer Modelle in Abhängigkeit ihres Gebrauchs im kommunikativen Kontext ein Desiderat für weitere theoretische und empirische Forschung bildet.

\section{LITERATURVERZEICHNIS}

[1] Adamzik, Kirsten (2004): Textlinguistik. Eine einführende Darstellung. Niemeyer: Tübingen.

[2] Antonovsky, Aaron (1979): Health, Stress and Coping: New Perspectives on Mental and Physical Well-Being. Jossey-Bass Publishers: San Francisco.

[3] Antonovsky, Aaron; Franke, Alexa (1997): Salutogenese: Zur Entmystifizierung der Gesundheit. dgtv-Verlag: Tübingen.

[4] Arntz, Rainer; Picht, Heribert; Mayer, Felix (2004): Einführung in die Terminologiearbeit. Olms: Hildesheim.

[5] Banse, Gerhard; Bechmann, Gotthard (1998): Interdisziplinäre Risikoforschung. Eine Bibliographie. Westdeutscher Verlag: Opladen. 
[6] Banse, Gerhard; Nelson, Gordon L; Parodi, Oliver (eds) (2011a): Sustainable Development - The Cultural Perspective. Concepts - Aspects - Examples. edition sigma: Berlin.

[7] Banse, Gerhard; Krebs, Irene (Hg.) (2011b): Kulturelle Diversität und Neue Medien. Entwicklungen, Interdependenzen, Resonanzen. trafo Wissenschaftsverlag, Berlin.

[8] Banse, Gerhard; Hauser, Robert; Machleidt, Petr; Parodi, Oliver (Hg.) (2013): Von der Informations- zur Wissensgesellschaft. e-Society, ePartizipation, e-Identität. edition sigma: Berlin.

[9] Beck, Ulrich (1986): Risikogesellschaft: Auf dem Weg in eine andere Moderne. Suhrkamp: Frankfurt.

[10] Beck, Ulrich (2007): Weltrisikogesellschaft. Suhrkamp: Frankfurt.

[11]BMBF (Bundesministerium für Bildung und Forschung) (2012): Zukunftsprojekt Erde (Wissenschaftsjahr 2012). (www.zukunftsprojekterde.de) (Zugriff 27.10.2013)

[12] Bonfadelli, Heinz (2012): Fokus Grüne Gentechnik: Analyse des medienvermittelten Diskurses. In: Weitze/Pühler (Hg.), 205-252.

[13] DUDEN 10 (2002): Bedeutungswörterbuch. Duden-Verlag: Mannheim.

[14]Fabry, Götz (2011): Vorlesung Medizinische Psychologie: Gesundheit und Krankheit; von der Patho- zur Salutogenese. (www.medpsych.unifreiburg.de/ skripts/salutogenese.pdf)

[15]Fischer, Andreas; Hahn, Gabriela (Hg.) (2001): Vom schwierigen Vergnügen einer Kommunikation über die Idee der Nachhaltigkeit. Frankfurt/Main.

[16]Franke, Alexa $\left(2006,2010^{2}\right)$ : Modelle von Gesundheit und Krankheit. Huber: Bern.

[17] Giebel, Daniela (2012): Integrierte Sicherheitskommunikation. Zur Herausbildung von Unsicherheitsbewältigungskompetenzen durch und in Sicherheitskommunikation. LIT Verlag: Berlin.

[18] Gill, Bernhard (2012): Utilitaristische Motive der Kritik - Warum die europäischen Bauern mehrheitlich nicht so begeistert von der Gentechnik sind. In: Weitze/Pühler (Hg.), 65 - 67.

[19] Grober, Ulrich (2010): Die Entdeckung der Nachhaltigkeit. Kulturgeschichte eines Begriffs. Kunstmann: München.

[20]Hansen, Klaus P. $\left(2004^{3}, 2011^{4}\right)$ : Kultur und Kulturwissenschaft. Eine Einführung. Francke (UTB): Tübingen.

[21]Hauser, Robert; Banse, Gerhard (2010): Kultur und Kulturalität. Annäherungen an ein vielschichtiges Konzept. In: Parodi et al (Hg.), 21-41.

[22] Hermann, Corinna (2009): Resilienz und systemisches Arbeiten in der psychosozialen Praxis.

[23] Hribal, Lucie (1999): Public Relations-Kultur und Risikokommunikation. Universitätsverlag: Konstanz.

[24] Hübner, Renate (2010): Die Magie der Dinge. Materielle Güter, Identität und Metaphysische Lücke. In Parodi et al., 2010 : 119-150.

[25]Hübner, Renate (2011): Sustainable Culture. A Contrast to an Efficiency Society? In: Banse et al., 205-221.

[26] Jacob, Karlheinz (1991): Maschine, mentales Modell, Metapher. Studien zur Semantik und Geschichte der Techniksprache. Niemeyer: Tübingen. 
[27] Johnson-Laird, Philip N (1983): Mental Models. Towards a Cognitive Science of Language. Cambridge University Press: Cambridge.

[28] Konerding, Klaus-Peter (1993): Frames und lexikalisches Bedeutungswissen. Niemeyer: Tübingen.

[29] Kopfmüller, Jürgen (2011): Von der kulturellen Dimension nachhaltiger Entwicklung zur Kultur nachhaltiger Entwicklung. In: Parodi et al., 43-57.

[30] Metzeltin, Michael (2007): Theoretische und angewandte Semantik. Vom Begriff zum Text. Praesens Verlag: Wien.

[31] Nielsen, Martin; Rittenhofer, Iris; Ditlevsen, Marianne G.; Pollach, Irene; Essmann Andersen, Sophie (Hg.) (2013 erscheint): Nachhaltigkeit in der Wirtschaftskommunikation. Verlag für Sozialwissenschaften: Wiesbaden.

[32] Parodi, Oliver; Banse, Gerhard; Schaffer, Axel (Hg.) (2010): Wechselspiele: Kultur und Nachhaltigkeit. Annäherungen an ein Spannungsfeld. edition sigma: Berlin.

[33]Regenfuß, Thomas; Viehweg, Klaus (2010): Sicherheits- und Risikoterminologie im Spannungsfeld von Technik und Recht. In: Winzer et al. (Hg.), S. 131- 144.

[34] Rentel, Nadine; Venohr, Elisabeth (Hg.) (2012): Text-Brücken zwischen den Kulturen. Lang: Frankfurt.

[35] Ropohl, Günter $\left(2009^{3}\right)$ : Allgemeine Technologie. Eine Systemtheorie der Technik. KIT-Verlag: Karlsruhe.

[36] Rothkegel, Annely (2008): Gebrauchstauglichkeit von Begriffen: Sicherheit, Risiko, Gefahr, Schutz. In: Forum Ware 2008, Wien.

[37] Rothkegel, Annely (2010a): Technikkommunikation. Produkte, Texte, Bilder. UTB 3214, UVK, Konstanz.

[38] Rothkegel, Annely (2010b): Sicherheitsmodelle und Kommunikationsrisiko. In: Winzer et al. (Hg.), 207-220.

[39] Rothkegel, Annely (2011b): Sicherheit durch Kommunikation: ein linguistischer Forschungsansatz. In: Banse/Krebs (Hg.), 269-284.

[40]Rothkegel, Annely (2012a): Umweltkommunikation in Europa: Schlüsselbegriffe im multilingualen Kontext (Nachhaltigkeit, Sicherheit, Resilienz). In: Zybatow/ Malgorzewicz (Hg.), 89-10.

[41] Rothkegel, Annely (2012b): Ränder und Übergänge in der fachorientierten Kommunikation. In: Rentel/Venohr (Hg.), 87-105.

[42] Rothkegel, Annely (2013a): Wissen im Web: Themen und Gemeinschaften. In: Banse et al. (Hg.), 63-81. trafo: Berlin.

[43] Rothkegel, Annely (2013b, erscheint): Zur Nachhaltigkeit von Text und Textarbeit. In: Nielsen et al.

[44] Schnotz, Wolfgang (1994): Aufbau von Wissensstrukturen. Beltz: Weinheim.

[45] Schiffer, Eckhard (2001): Wie Gesundheit entsteht - Salutogenese: Schatzsuche statt Fehlerfahndung. Beltz: Göttingen.

[46] Schubert, Klaus (2007): Wissen, Sprache, Medium, Arbeit. Ein integratives Modelle der ein- und mehrsprachigen Fachkommunikation. Narr: Tübingen.

[47] Siegrist, Ulrich (2009): Coaching in Krisen. Resilienzkonzepte in der Praxis. In: Zeitschrift für Gesprächspsychotherapie und Personenzentrierte Beratung 4/2009, 199-205. 
[48] Stieniczka, Norbert (2006): Das 'narrensichere' Auto. Die Entwicklung passiver Sicherheitstechnik in der Bundesrepublik Deutschland. Wissenschaftliche Buchgesellschaft: Darmstadt.

[49] Thoma, Klaus; Drees, Birgit; Leismann, Tobias (2010): Zukunfstechnologien in der Sicherheitsforschung. In: Winzer et al. (Hg.),13-37.

[50] Trabant, Jürgen (2012): Weltansichten. Wilhelm von Humboldts Sprachprojekt. Beck: München.

[51]Trachsler, Daniel (2009): Resilienz - Konzept zur Krisen- und Katastrophenbewältigung. In: CSS Analysen zur Sicherheitspolitik, Nr. 60, 13. (www.css.ethz.ch)

[52] Weber, Jutta (Hg.) (2010): Interdisziplinierung? Zum Wissenstransfer zwischen den Geistes-, Sozial- und Technowissenschaften. transcript: Bielefeld.

[53] Weitze, Marc-Denis; Pühler, Alfred; Heckl, Wolfgang M.; Müller-Röber, Bernd; Renn, Ortwin; Weingart, Peter; Wess, Günther (Hg.) (2012): Biotechnologie-Kommunikation. Kontroversen, Analysen, Aktivitäten. acatech Diskussion. Springer Viehweg: Berlin, Heidelberg.

[54] Winzer, Petra; Schnieder, Eckehard; Bach, Friedrich-Wilhelm (Hg.) (2010): Sicherheitsforschung - Chancen und Perspektiven. acatech diskutiert. Springer: Berlin.

[55]Ziem, Alexander (2008): Sprachliches Wissen und Frames. Kognitive Aspekte der semantischen Kompetenz. de Gruyter: Berlin.

[56]Zwack, Julia (2013): Resilienz im Arztberuf. Studie an der Universitätsklinik Heidelberg (www.oberbergkliniken.de) Zugriff 27.10.2013.

[57]Zybatow, Lew N.; Malgorzewicz, Anna (Hg.) (2012): Sprachenvielfalt in der EU und Translation, Studia Translatorica 3, Neisse Verlag: Dresden.

\section{RISK AND RESILIENCE WITHIN COMMUNICATION AROUND SUSTAINABLE DEVELOPMENT: SEMANTIC MODELS IN DEALING WITH CRISES}

The purpose of this article was to discuss some fundamental issues around the semantic discourses on the sustainable development based upon the selected theoretical models of risk and resilience. The starting point of the considerations are the thematic analysis of authentic texts (print and online) within the use and meaning of the concepts of risk and resilience, which on the one hand show some aspects of the risks, and on the other one they feature possible strategies for overcoming the crisis. The characteristics of communication about sustainable development are weave threads from different disciplines and confusing technical language with every day one. Experts and non-expert help to resolve substantive issues (such as the financial crisis, back in power industry, protection of biodiversity, climate change), which overlap with communication problems. The latter is a challenge to the linguistic discourse analysis are desideratum for theoretical and empirical research.

Based on the selected models of risk and resilience models it was shown how identification of semantic models in terms of different patterns of understanding and interpretation helps to reveal possible problems in communication. And because the main part of the communication around sustainable development is conceived as a crisis prevention, the focus was put on the specialized concepts of risk and resilience that in everyday communication will be used relatively infrequently, even though these words are not entirely unknown. 
Risk communication models are essentially dependent on the individual disciplines (e.g. risk of danger / risk of injury / loss, safety / confidence, gambling / gain), while as to the concept of resilience it refers only partially (e.g. the ability to stop, regeneration capacities, mental stability, balance, strength (technical systems)). At this moment the universal assignments have created that correspond to the higher-level model of health (in the broadest sense). While risk models cover primarily general social framework for communication about sustainable development, thus the resilience models are more related to the individual.

Keywords: risk, resilience, semantic analysis, social communication, sustainable development, crisis management.

\section{RYZYKO I REZYLIENCJA W RAMACH KOMUNIKACJI WOKÓŁ ZRÓWNOWAŻONEGO ROZWOJU: MODELE SEMANTYCZNE W OBCOWANIU Z KRYZYSAMI}

Przedłożony przyczynek próbuje na podstawie wybranych teoretycznych modeli ryzyka i rezyliencji pociagnąć kilka fundamentalnych linii przez terytorium semantyczne dyskursów wokół zrównoważonego rozwoju. Punkt wyjścia rozważań stanowią tematyczne analizy autentycznych tekstów (drukowanych i online) pod kątem sposobów użycia i znaczeń pojęć ryzyka i rezyliencji, które z jednej strony znamionują aspekty zagrożeń, z drugiej zaś możliwe strategie przezwyciężania kryzysów. Cechami charakterystycznymi komunikacji wokół zrównoważonego rozwoju są splot tematów pochodzących z różnych dyscyplin oraz pomieszanie języka specjalistycznego i potocznego. Eksperci i nieeksperci uczestniczą w rozwiązywaniu merytorycznych problemów (np. kryzysu finansowego, zwrotu w energetyce, ochrony bioróżnorodności, zmian klimatycznych), na które nakładają się problemy komunikacyjne. Te ostatnie są wyzwaniem dla lingwistycznej analizy dyskursu i stanowią dezyderat dla badań teoretycznych i empirycznych.

$\mathrm{Na}$ podstawie wybranych modeli ryzyka resp. modeli rezyliencji egzemplarycznie zademonstrowano, jak identyfikacja semantycznych modeli w sensie odmiennych wzorców rozumienia i interpretacji pomaga odsłonić możliwe problemy w porozumiewaniu się. A ponieważ główną część komunikacji wokół zrównoważonego rozwoju pojmuje się jako zapobieganie kryzysom, wybór padł na specjalistyczne pojęcia ryzyka i rezyliencji, które w codziennej komunikacji z pewnością używane są stosunkowo rzadko, choć przecież słowa te nie są całkowicie nieznane.

Modele komunikacji ryzyka są w gruncie rzeczy zależne od poszczególnych dyscyplin (np. ryzyko z perspektywy niebezpieczeństwa/zagrożenia, szkody/straty, bezpieczeństwa/pewności, hazardu/zysku), podczas gdy do pojęcia rezyliencji odnosi się to tylko częściowo (np. zdolności do oporu, zdolności regeneracyjne, psychiczna stabilność, równowaga, wytrzymałość (systemy techniczne)). Tutaj wykrystalizowały się uniwersalne przyporządkowania, które odpowiadają nadrzędnemu modelowi zdrowia (w najszerszym sensie). Podczas gdy modele ryzyka pokrywają głównie ogólnospołeczne ramy komunikacji wokół zrównoważonego rozwoju, modele rezyliencji bardziej odnoszą się do jednostki.

Słowa kluczowe: ryzyko, rezyliencja, analiza semantyczna, komunikacja społeczna, zrównoważony rozwój, zarządzanie kryzysowe.

DOI:10.7862/rz.2013.hss.19

Tekst złożono w redakcji: kwiecień 2013

Przyjęto do druku: wrzesień 2013 